\title{
Domain structures and local switching in lead-free piezoceramics
}

\section{$\mathrm{Ba}_{0.85} \mathrm{Ca}_{0.15} \mathrm{Ti}_{0.90} \mathrm{Zr}_{0.10 \mathrm{O}_{3}}$}

A. P. Turygin' , M. M. Neradovskiy, N. A. Naumova, D. V. Zayats, I. Coondoo, A. L. Kholkin, and V. Ya. Shur

Citation: Journal of Applied Physics 118, 072002 (2015); doi: 10.1063/1.4927802

View online: http://dx.doi.org/10.1063/1.4927802

View Table of Contents: http://aip.scitation.org/toc/jap/118/7

Published by the American Institute of Physics

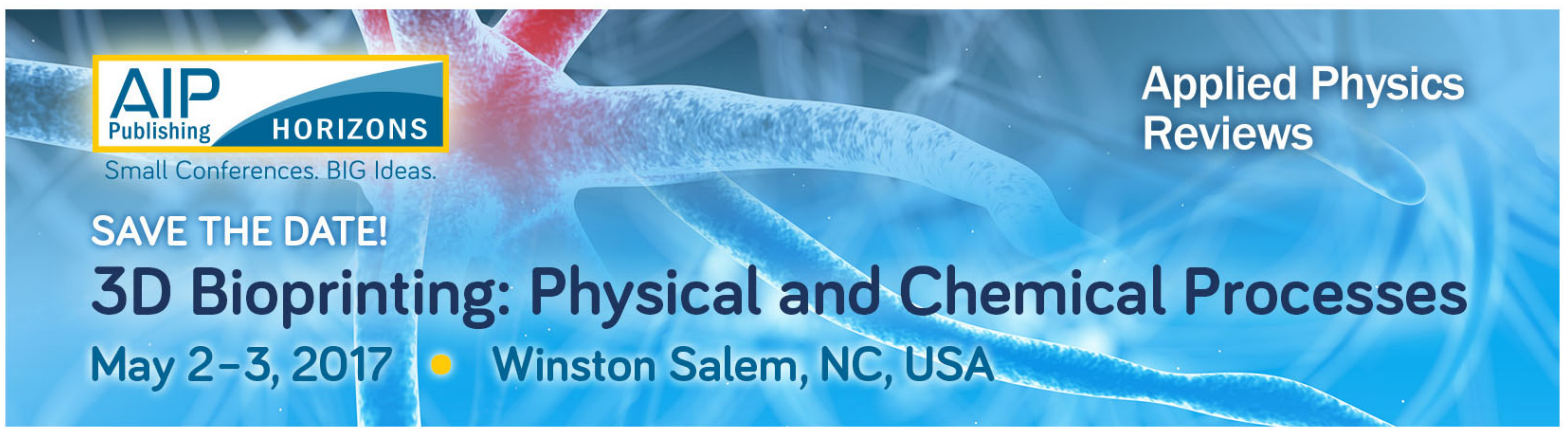




\title{
Domain structures and local switching in lead-free piezoceramics $\mathrm{Ba}_{0.85} \mathrm{Ca}_{0.15} \mathrm{Ti}_{0.90} \mathrm{Zr}_{0.10} \mathrm{O}_{3}$
}

\author{
A. P. Turygin, ${ }^{1, \text { a) }}$ M. M. Neradovskiy, ${ }^{1}$ N. A. Naumova, ${ }^{1}$ D. V. Zayats, ${ }^{1}$ I. Coondoo, ${ }^{2}$ \\ A. L. Kholkin, ${ }^{1,2}$ and V. Ya. Shur ${ }^{1}$ \\ ${ }^{1}$ Institute of Natural Sciences, Ural Federal University, 620000 Ekaterinburg, Russia \\ ${ }^{2}$ Department of Physics \& CICECO Aveiro Institute of Materials, University of Aveiro, \\ 3810193 Aveiro, Portugal
}

(Received 23 November 2014; accepted 24 March 2015; published online 19 August 2015)

\begin{abstract}
Lead-free piezoelectrics are becoming increasingly important in view of environmental problems of currently used lead-based perovskites such as lead zirconate titanate (PZT). One of the recent candidates for PZT replacement, solid solutions of $\mathrm{BaZr}_{0.2} \mathrm{Ti}_{0.8} \mathrm{O}_{3}$ and $\mathrm{Ba}_{0.7} \mathrm{Ca}_{0.3} \mathrm{TiO}_{3}$, are investigated in this work by piezoresponse force microscopy. Coexistence of the tetragonal and rhombohedral phases in this material is observed, which probably gives rise to easy polarization switching due to multiple domain states. The period of observed domain lamella scales with the grain size obeying well-known square root dependence characteristic of $\mathrm{BaTiO}_{3}$ ceramics. Domain switching and relaxation are investigated at the nanoscale as a function of the applied voltage and duration of the applied voltage pulses. The observed distortion of piezoresponse hysteresis loops near grain boundaries is attested to the increased concentration of defects. Nanoscale piezoelectric properties of these materials are discussed. (C) 2015 AIP Publishing LLC.
\end{abstract}

[http://dx.doi.org/10.1063/1.4927802]

\section{INTRODUCTION}

Piezoelectric materials are intensively used in sensors and actuators due to their extremely high electromechanical coupling coefficients and an ability to work under high mechanical stress. ${ }^{1}$ Compared with other materials for sensing and actuation, piezoelectric materials can work at high frequencies and temperatures, are chemically stable, and can be scaled down for use in micro- and nanoelectromechanical systems. $^{2}$ The fundamental issue of loss of spontaneous polarization (thus piezoelectricity) due to the large surface energy that can cause piezoelectric crystalline structures to be thermodynamically unstable in ultrathin films with a few-unit-cell thickness has been discussed by various authors. ${ }^{3-5}$ On the contrary, synchrotron X-ray study of lead titanate films as thin as a single unit cell showed that the ferroelectric phase is stable for thicknesses down to 3 unit cells $(1.2 \mathrm{~nm})$ at room temperature. ${ }^{6}$

Wide use of piezoelectric materials is currently prevented by their essential component lead, which presents significant environmental and ecological concerns as hazard material. Efforts of many research groups are currently focused on the replacement of the most popular piezoelectric materials lead zirconate titanate, $\mathrm{Pb}\left(\mathrm{Zr}_{\mathrm{x}} \mathrm{Ti}_{1}{ }_{\mathrm{x}}\right) \mathrm{O}_{3}$ (PZT), with other piezoelectrics having similar performance but without lead. In this context, several lead-free piezoelectric systems have been studied such as $\mathrm{K}_{0.5} \mathrm{Na}_{0.5} \mathrm{NbO}_{3}(\mathrm{KNN})$, $\mathrm{Na}_{0.5} \mathrm{Bi}_{0.5} \mathrm{TiO}_{3}$ (NBT), and $\mathrm{BaTiO}_{3}$ (BT) based solid solutions. ${ }^{7,8}$ Although the piezoelectric performance of KNN and NBT systems have been improved using site engineering, ${ }^{9,10}$ sintering aid, ${ }^{11}$ or novel synthesis method, ${ }^{12}$ their main

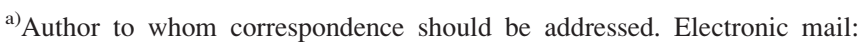
anton.turygin@labfer.usu.ru
}

disadvantages (presence of highly volatile elements $\mathrm{Bi}, \mathrm{Na}$, and $\mathrm{K}$ and hydroscopicity) still remain.

Recently, the strain driven morphotropic phase boundary (MPB) in $\mathrm{BiFeO}_{3}$ films has been of great interest as it potentially possesses a giant polarization $\left(\sim 150 \mu \mathrm{C} / \mathrm{cm}^{2}\right)$ and much enhanced electromechanical response. ${ }^{13-15}$

Another perspective materials for PZT replacement is a binary system of barium zirconate-titanate barium-calcium titanate solid solution (BZT-BCT), which does not possess any volatile elements and is not hydroscopic. BZT-BCT ceramics were reported to exhibit high dielectric constant ${ }^{16}$ and significantly elevated longitudinal piezoelectric coefficient $\left(\mathrm{d}_{33} \approx 620 \mathrm{pC} / \mathrm{N}\right) .{ }^{17}$ Phase diagram of $(1-\mathrm{x}) \mathrm{BZT}$ $\mathrm{xBCT}$ system is similar to $\mathrm{Pb}$-based systems, with a MPB starting from a triple point of the paraelectric cubic, ferroelectric rhombohedral, and tetragonal phases. ${ }^{18}$ This triple point is shown to be a tricritical point, and, therefore, the system exhibits very high piezoelectric performance. Transmission Electron Microscopy (TEM) study $^{19}$ has shown that there is a link between the piezoelectric activity and domain assemblages in this material. It was found that ferroelectric domains become miniaturized down to a nanometer size with apparent domain hierarchy that is coincident with the $\mathrm{d}_{33}$-maximum region on the phase diagram. First Piezoresponse Force Microscopy (PFM) study ${ }^{20}$ revealed a complex mosaic-like domain structure with sub- $\mu \mathrm{m}$ domain periodicity and clear nanoscale polarization switching as documented by the nanoscale piezoresponse hysteresis loops. This work is focused on further study of the 50 BZT 50BCT lead-free system using nanoscale piezoelectric methods. Domain assemblages and their behavior under localized electric field provided by the sharp tip of PFM are investigated as a function of time and duration of poling pulse. The 


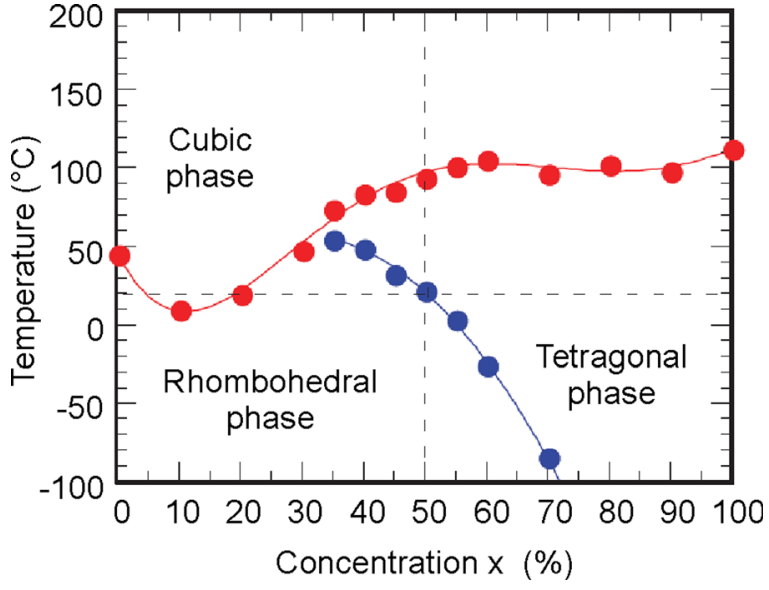

FIG. 1. Phase diagram for BZT BCT system (adapted from Ref. 21).

evolution of the hysteresis loops is studied as a function of the position inside the grain.

\section{EXPERIMENTAL}

Ceramics of the composition $\mathrm{Ba}_{0.85} \mathrm{Ca}_{0.15} \mathrm{Ti}_{0.90} \mathrm{Zr}_{0.10} \mathrm{O}_{3}$ (BCZT) were prepared by the conventional solid-state reaction technique in which stoichiometric ratios of $\mathrm{BaCO}_{3}$ (99.9\%), $\mathrm{CaCO}_{3}$ (99\%), $\mathrm{TiO}_{2}$ (99\%), and $\mathrm{ZrO}_{2}(99 \%)$ were mixed. Calcination was performed at $1200^{\circ} \mathrm{C}$ for $6 \mathrm{~h}$. The calcined powder was then pressed into pellets (using polyvinyl alcohol as a binder) and sintered at $1450{ }^{\circ} \mathrm{C}$ for $2 \mathrm{~h}$ in air. Pure perovskite phase formation was confirmed by X-ray diffraction (XRD, PhillipsX'pert, the Netherlands). The studied composition exhibits phase transition between rhombohedral and tetragonal phases (morphotropic phase boundary) at room temperature (Fig. 1). ${ }^{21}$ Detailed procedure of the samples preparation and their structural characterization can be found elsewhere. ${ }^{20}$ The grain size in the BCZT samples was in the range from 4 to $18 \mu \mathrm{m}$. Ceramic pellets were polished to optical quality by using diamond paste and cleaned in alcohol and acetone afterwards.

Local polarization study was performed using PFM with Probe Nanolaboratory NTEGRA Aura (NT-MDT, Russia). NI-6251 multifunction Data Acquisition board (DAQ) and high-voltage amplifier Trek-677B were used for the application of DC voltage. We used commercial probes ASYLEC-01 with titanium-iridium conductive coating (Asylum Research) with a radius of curvature $\mathrm{R}=28 \mathrm{~nm}$ and resonance frequency $\mathrm{f}=70 \mathrm{kHz}$ and spring constant $\mathrm{k}=2 \mathrm{~N} / \mathrm{m}$. Other types of probes were used as well: NSG01 coated with tungsten carbide coating $(\mathrm{R}=10 \mathrm{~nm}, \mathrm{f}=150 \mathrm{kHz}, \mathrm{k}=5.1 \mathrm{~N} / \mathrm{m})$ and
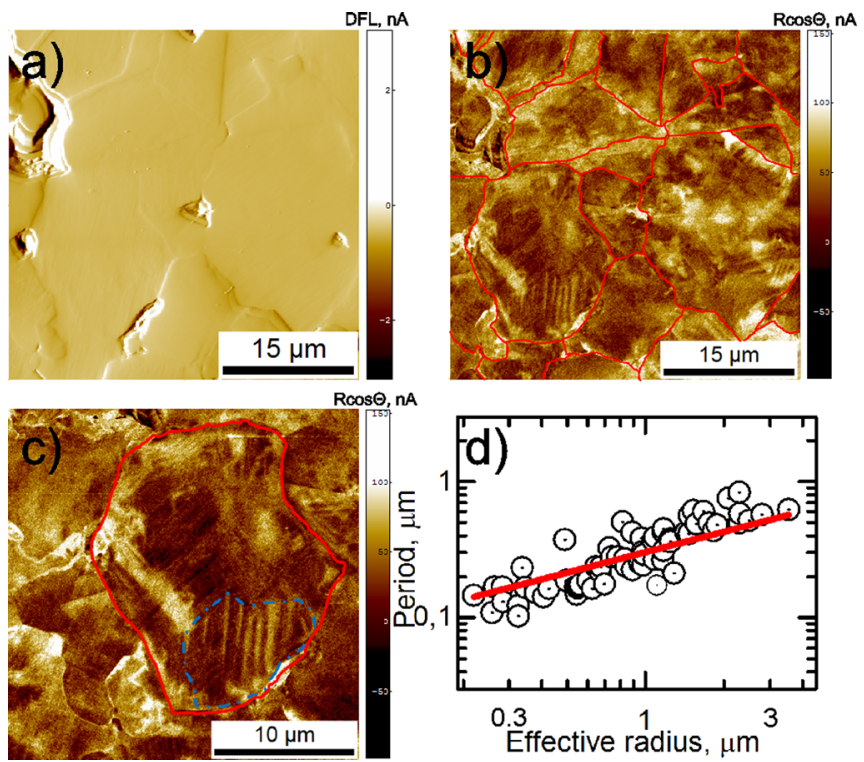

FIG. 3. (a) Deflection signal of BCZT surface, (b) corresponding PFM image of several grains with grain boundaries outlined by red line, (c) indi vidual grain with a subgrain filled by domain lamella (blue dotted line), (d) domain period as a function of subgrain radius. Experimental points are fit ted with Eq. (1).

NSG20 coated with tungsten carbide coating $(\mathrm{R}=10 \mathrm{~nm}$, $\mathrm{f}=420 \mathrm{kHz}, \mathrm{k}=48 \mathrm{~N} / \mathrm{m}$ ), both from NT-MDT. No significant difference in obtained results was found. For the piezoresponse acquisition, we applied $\mathrm{AC}$ voltage of $35 \mathrm{~V}$ amplitude both at the frequencies above and below resonance at the plateau of the piezoresponse frequency dependence. Localized switching was performed by the application of DC bias pulses to the same location inside the grain, so that the memory effect could be present in switching experiments.

\section{RESULTS AND DISCUSSION}

\section{A. As-grown domain structure}

Typical domain structure of a BCZT unpoled ceramic grain is shown in Figs. 2 and 3 along with the grain topography. Complex domain structure consisting of multiple domain patterns (including periodic lamella) was found (Fig. 2(b)). This confirms high quality of the ceramics as there are no amorphous areas without piezoresponse (either in out-of-plane or in-plane modes). We suggest that these domain states reflect the coexistence of tetragonal and rhombohedral phases in which the observed domain lamella could represent well-known tetragonal or rhombohedral twins formed due to minimization of elastic energy
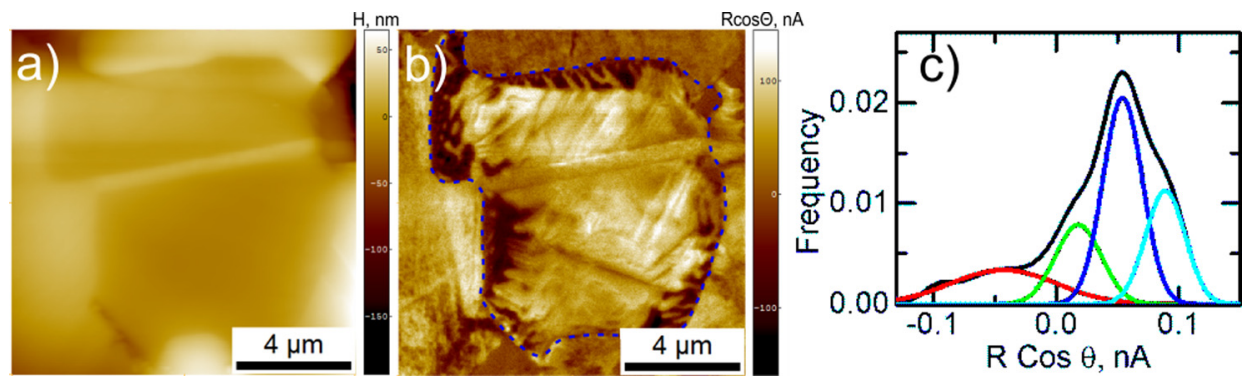

FIG. 2. (a) Topography of the polished surface of a BCZT sample, (b) PFM image $(R \cos \theta)$, (c) histogram of the PFM signal in a grain marked by dashed line. Fitting with Gaussians was done to deconvolute four peaks corresponding to different domain states in (b). 
associated with the tetragonal/rhombohedral distortion. ${ }^{22}$ It is interesting to note that the observed twins are mostly concentrated near the grain boundaries. This behavior can be rationalized in terms of higher stress developed at the grain boundaries, which requires higher concentration of twins to relieve the stress. The histogram of the piezoresponse image of the individual grain (Fig. 2(c)) is a signature of the domain states with four distinct peaks (after deconvolution with overlapped Gaussian peaks ${ }^{23}$ ). These peaks apparently represent preferred domain states characteristic of the given orientation and crystallographic symmetry of the studied grain. The presence of multiple domain states hints to the possible phase coexistence in BCZT ceramics in line with the recent observation of the complex hierarchical domain structure by TEM. ${ }^{18}$ The averaged domain periods of lamella patterns were then extracted by using a Fourier transform as a function of the corresponding "subgrain" radius (we suppose that each subgrain represents either tetragonal or rhombohedral phase within the individual ceramic grain) (Fig. 3(c)). In order to understand the grain size dependence in BCZT ceramics, we plotted the average period of the domain structure as a function of the effective radius of the corresponding subgrain area (calculated as $R_{e f f}=\sqrt{\frac{A}{\pi}}$, where A is the subgrain area). Statistical analysis of the periodic domain structures (Fig. 3(d)) demonstrates that, on average, the obtained dependence is close to well-known square root dependence of the domain period $(d)$ as a function of the effective radius of the subgrain

$$
d\left(R_{e f f}\right)=b \sqrt{ } R_{e f f},
$$

where $b=\sqrt{\frac{128 \pi \sigma \sigma}{C_{11} S_{s}^{2}}}, \sigma$ is the domain energy, $C_{11}$ is the longitudinal elastic constant, $S_{\mathrm{S}}=(1-a / c)$ is the spontaneous strain, and $a$ and $c$ are the lattice parameters.

This dependence has been first derived by Arlt et al. ${ }^{24}$ and then has been widely used for the explanation of the dielectric and piezoelectric properties of $\mathrm{BaTiO}_{3}$-based ferroelectric ceramics. ${ }^{25,26} \mathrm{We}$ thus deduce that the mechanism of twin formation is similar to that of conventional ferroelectric perovskite ceramics but the interphase boundaries play the role of grain boundaries in the case of solid solutions with coexisting tetragonal and rhombohedral phases. The phase coexistence and motion of the interphase boundaries (often facilitated by the transitory monoclinic phase ${ }^{27}$ ) may contribute to the high macroscopic piezoresponse of these ceramics. It should be noted that domain wall clamping may limit the piezoresponse as recently observed in ferroelectric films. ${ }^{28,29}$ The reported parameters of BCZT ceramics $^{30,31}$ are as follows: $C_{11}=13.6 \times 10^{10} \mathrm{~N} / \mathrm{m}^{2}, S_{\mathrm{S}}$ $=0.0053, a=3.9963 \AA$, and $c=4.0175 \AA$. By substituting these values into Eq. (1), we can obtain the average domain wall energy in BCZT ceramics $\sigma=1.0 \pm 0.63 \mathrm{~mJ} / \mathrm{m}^{2}$. This value is not far from the classical estimation by Zhirnov ${ }^{32}$ $\left(3 \mathrm{~mJ} / \mathrm{m}^{2}\right)$ and close to recent theoretical calculations of domain energy in $\mathrm{BaTiO}_{3} .{ }^{33}$ The difference is expected for the BCZT solid solutions with their intrinsic disorder and presence of multiple domain states. It should be noted that the classical parabolic law is obeyed well below the limit of $\sim 1 \mu \mathrm{m}$ (as compared to single phase $\mathrm{BaTiO}_{3}{ }^{24}$ ). It may be related to different boundary conditions and stress states at the grain and interphase boundaries.

\section{B. Local switching and domain wall motion}

Local switching was performed in single grains by the application of a sequence of DC voltage pulses with variable amplitude $(10200 \mathrm{~V})$ and duration $(0.210 \mathrm{~s})$ to the conductive tip. The scanning procedure was done in between the pulses allowing visualization of the resulting domain structure. This type of measurements was first introduced by Tybell et al $^{34}$ to study domain wall motion in PZT thin films. More recently, the method was also used to investigate the domain wall motion in $60 \mathrm{~nm} \mathrm{BaTiO}_{3}$ films $^{35}$ and bulk PLZT the ceramics. ${ }^{36}$ Switching pulses produced sufficiently stable circular domains (Fig. 4), in which the diameter depended on the pulse amplitude and duration. In addition, we observed that the central part of the circular domain shows a bright (opposite) contrast thus forming two concentric (nested) domains of the entire structure (Fig. 4(b)). Such behavior (called anomalous polarization inversion ${ }^{37}$ ) has been frequently observed in local switching experiments ${ }^{38-41}$ and was attested to the charge injection either into the bulk ${ }^{37}$ or surface of the samples. ${ }^{40}$

First, we consider the voltage dependence of the diameter of induced domain under high voltages. It is essentially non-linear hinting to the significant slowing down of the domain velocity at large distances from the tip. Fitting with the known equations for the domain wall motion under the action of the inhomogeneous electric field produced by the PFM tip (see Ref. 35 and references therein) did not give satisfactory results. We note that the measurement conditions (application of the voltages to the same point) could significantly change the domain propagation mechanism due to substantial pinning and stabilization of domain walls by bulk screening. Another scenario could be related to the conductivity of the absorbate layer, which effectively screens the polarization of growing domain. ${ }^{42}$

The logarithmic dependence of switched domain radius on pulse duration is observed only for sizes above $1 \mu \mathrm{m}$ (Fig. 5(c)). Similar behavior has been found in congruent $\mathrm{LiNbO}_{3}$ doped with $\mathrm{MgO}{ }^{42}$ The observed behavior was attributed to the existence of the surface layer with low conductivity which changes drastically the spatial distribution of

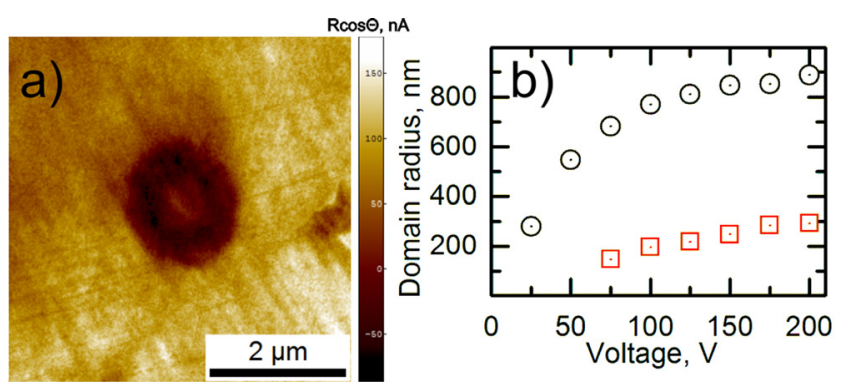

FIG. 4. (a) PFM image of the polarized area switched under a DC pulse of $200 \mathrm{~V}$ applied for $5 \mathrm{~s}$. (b) Voltage dependences of the external (circles) and internal (squares) radii of induced domains in BCZT ceramics. 

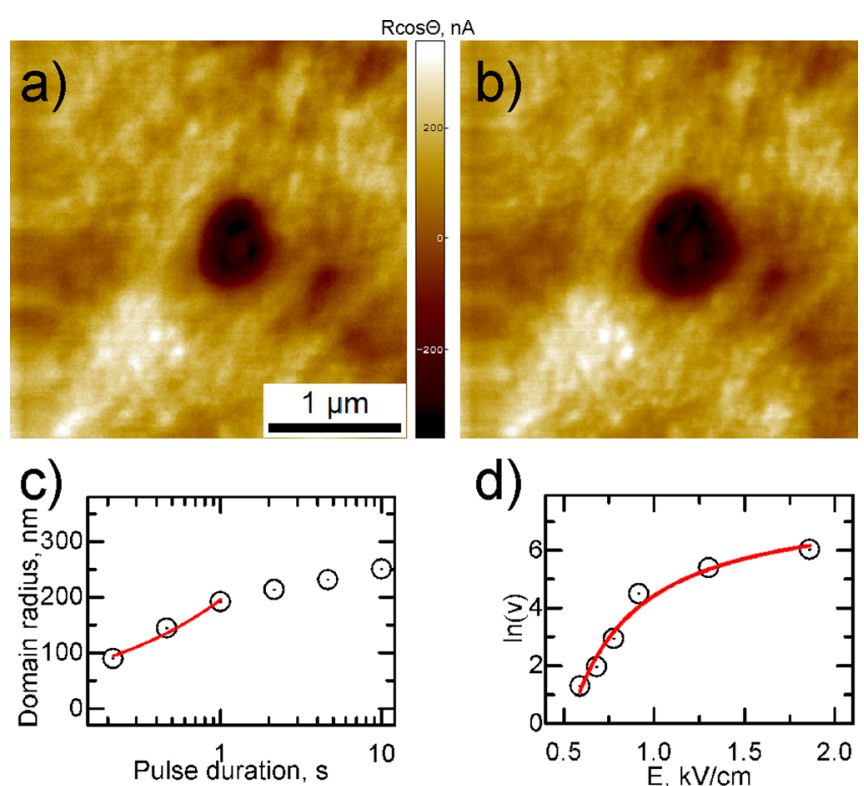

FIG. 5. PFM images of domains polarized by DC pulses: (a) $20 \mathrm{~V}, 1 \mathrm{~s}$ and (b) $20 \mathrm{~V}, 10 \mathrm{~s}$. (c) Dependence of domain radius on pulse duration fitted by Eq. (2). (d) Field dependence of the domain wall velocity fitted by Eq. (3).

the switching electric field. In addition to a strongly localized field produced by the tip, there exists homogeneous field which propagates over the large distance from the tip. The existence of the adsorbed surface layer leads to a so-called "current limited switching process," growth is controlled by the current in external circuit including low conducting layer. The wall motion in this homogeneous field is possible only in case of efficient enough screening of the depolarizing field. The proposed quasi-static description of the isolated domain growth accounting for the retardation of the depolarizing field screening was able to explain the dependences of the domain radius on switching pulse duration. ${ }^{41}$ It was shown experimentally that that the sample surface is covered by thin water layer for switching in ambient conditions. ${ }^{43,44}$ Thus, the obtained time dependences of the domain sizes depend significantly on the humidity. $^{45,46}$ Following this approach, we rationalized the evolution of the domain radius with time in our case as

$$
r(U, t)=a U+b t^{0.5}-c
$$

where $U$ is the applied voltage, $a=\frac{1}{2 K E_{d e p}}, b=\left(\frac{I}{\varepsilon \varepsilon_{o} \pi} \frac{1}{E_{d e p}}\right)^{0.5}$, $c=\frac{d}{2 K} \frac{E_{t h}}{E_{d e p}}, K$ is the depolarizing factor, $E$ is the depolarizing field, $\varepsilon$ is the dielectric permittivity, $\varepsilon_{\mathrm{o}}$ is the permittivity of vacuum, $d$ is the thickness of the sample, $E_{\mathrm{th}}$ is the threshold field, and $I=$ const is a switching current.

Fitting of our experimental data with Eq. (2) demonstrates an abrupt change to the logarithmic time dependence for pulse duration above $1 \mathrm{~s}$ (Fig. 5(c)). Similar behavior obtained for congruent $\mathrm{LiNbO}_{3}$ doped with $\mathrm{MgO}$ has been attributed to the transition from stochastic to determined nucleation induced by retardation of bulk screening. ${ }^{41}$

Obtained experimental data (Fig. 5(c)) allowed us to extract the field dependence of the domain wall motion velocity (Fig. 5(d)). The field value at the domain wall has been calculated in the point charge approximation. ${ }^{40}$ The obtained field dependence of velocity was fitted (Fig. 5(d)) with the activation law taking into account the existence of internal bias field

$$
v(E)=v_{0} \exp \left(-\frac{E_{a c}}{E-E_{b}}\right),
$$

where $E_{\mathrm{ac}}$ is the activation field, $E_{\mathrm{b}}$ is the internal bias field, and $v_{0}$ is the parameter.

Fitting gave the following values for the involved parameters: $E_{\mathrm{ac}}=2.8 \mathrm{kV} / \mathrm{cm}$ and $E_{\mathrm{b}}=0.17 \mathrm{kV} / \mathrm{cm}$. The obtained results match the typical values for bulk ferroelectrics (e.g., for near-stoichiometric $\mathrm{LiTaO}_{3}, E_{\mathrm{ac}}=5.34 \mathrm{kV} / \mathrm{cm}$ and $E_{\mathrm{b}}$ $=2.15 \mathrm{kV} / \mathrm{cm}$ (Ref. 47) and for stoichiometric $\mathrm{LiTaO}_{3}$ produced by vapor transport equilibration, $E_{\mathrm{ac}}=10.8 \mathrm{kV} / \mathrm{cm}$ and $E_{\mathrm{b}}=0.08 \mathrm{kV} / \mathrm{cm}$, Ref. 48 ).

It is interesting to note that the absolute values of the apparent domain wall velocity (Fig. 5(d)) are at least one order of magnitude higher than in $\mathrm{BaTiO}_{3}$ films ${ }^{35}$ and PLZT ceramics. ${ }^{36}$ This can be, in principle, the reason for the superior piezoelectric performance of BCZT ceramics.

\section{Domain relaxation}

It was found that the produced domain patterns written by the moving biased tip are not stable and slowly disappear. This happened because the poling time experienced by every pixel is less than that required for the creation of a stable domain under the PFM tip. ${ }^{49}$ This allowed us to qualitatively study the backswitching process in detail. Square domain pattern (Fig. 6) was created by scanning a $3 \times 3 \mu \mathrm{m}^{2}$ area under $+10 \mathrm{~V}$. After that a smaller square pattern of $1.5 \times 1.5 \mu \mathrm{m}^{2}$ in size was created using negative bias of $-10 \mathrm{~V}$. The domain images were acquired each $15 \mathrm{~min}$ by scanning the entire area with AC voltage of $3 \mathrm{~V}$ amplitude. The structure evolves
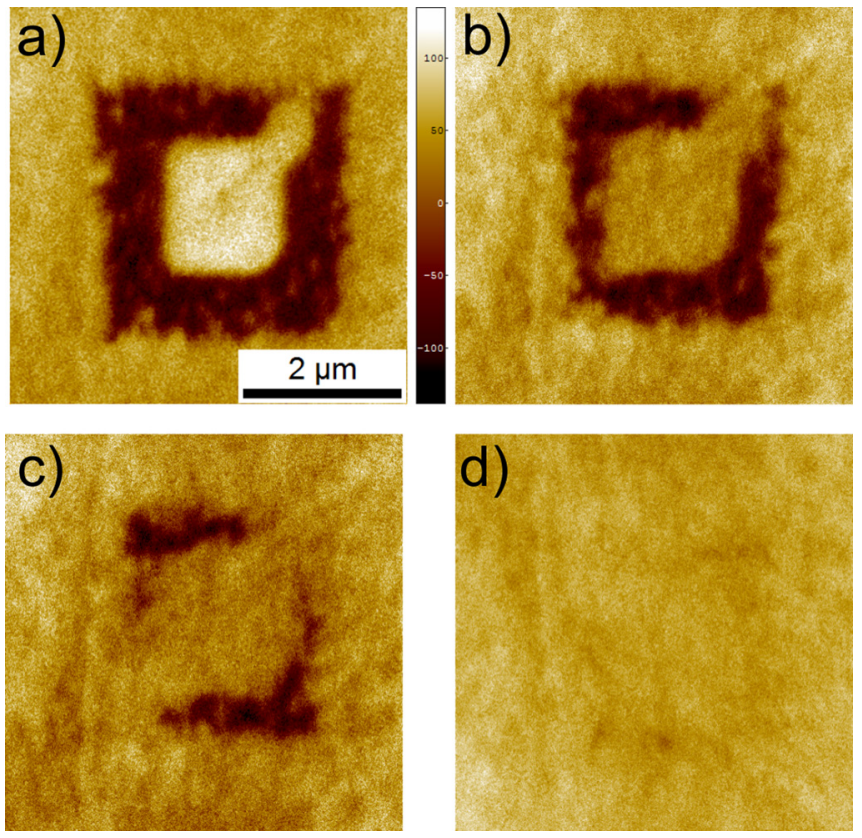

FIG. 6. Time relaxation of the produced domain pattern. PFM images after (a) $2 \mathrm{~min}$, (b) $95 \mathrm{~min}$, (c) $168 \mathrm{~min}$, and (d) $294 \mathrm{~min}$. 

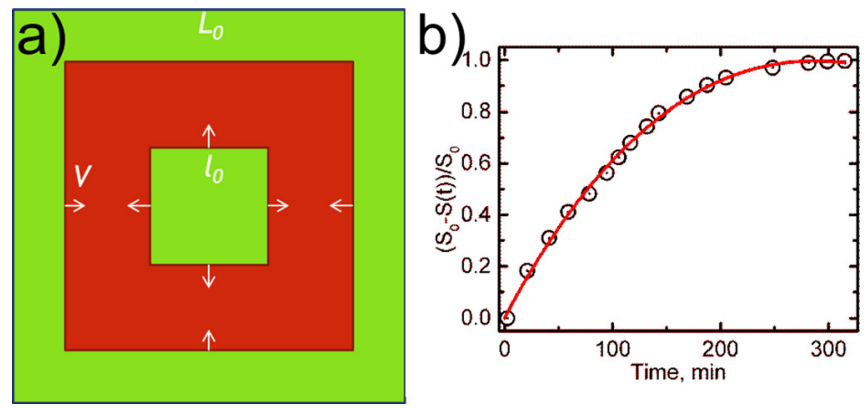

FIG. 7. (a) Schematic of the domain pattern backswitching. (b) Time de pendence of the normalized domain area. Experimental points are fitted by Eq. (8).

significantly with time and disappeared completely after several hours (Fig. 6). For the analysis of the domain relaxation, we have used a depolarization field model extensively studied in Refs. 50 and 51.

We have considered the relaxation process as a result of domain evolution under the action of a backswitching field $E_{\mathrm{bs}}$, which corresponds to the value of the residual depolarizing field exponentially decreasing with time due to bulk screening process ${ }^{52}$

$$
E_{b s}(t)=E_{r d}(t) e^{\frac{t}{\tau}}
$$

where $\tau$ is the characteristic time of screening process.

If the external and internal borders of the embedded square pattern move independently towards each other, the area of black domain decreases as a function of time (Fig. 7)

$$
S(t)=L(t)^{2}-l(t)^{2}
$$

where $L(t)$ and $l(t)$ are the linear sizes of the external and internal squares, respectively.

For linear field dependence of the domain wall velocity, the following equations apply:

$$
\begin{gathered}
v(E, t)=\mu\left(E_{r d} e^{\frac{t}{\tau}}-E_{t h}\right), \\
S(t)=S_{0}-4 \mu\left(L_{0}-l_{0}\right)\left(E_{r d} \tau\left(1-e^{\frac{t}{\tau}}\right)-E_{t h} t\right),
\end{gathered}
$$

where $S_{0}=L_{0}^{2}-l_{0}^{2}$ is the initial area of square pattern.

The fractional change of the pattern area is therefore

$$
\frac{\Delta S}{S_{0}}(t)=\frac{4 \mu}{\Delta L}\left(E_{r d} \tau\left(1-e^{\frac{t}{\tau}}\right)-E_{t h} t\right),
$$

where $\Delta L=L_{0}-l_{0}$.

Fitting experimental data of Fig. 7(b) with Eq. (8) allowed extracting important parameters characterizing the backswitching process: $E_{t h}=0.7 \mathrm{kV} / \mathrm{cm}, E_{r d}=1.47 \mathrm{kV} / \mathrm{cm}$, $\mu=6.4 \times 10{ }^{16} \mathrm{~m}^{2} / \mathrm{V} \cdot \mathrm{s}$, and $\tau=380 \mathrm{~min}$. Similar values of the relaxation time were obtained in $\mathrm{Pb}_{5} \mathrm{Ge}_{3} \mathrm{O}_{11}$ and $\mathrm{Gd}_{2}\left(\mathrm{MoO}_{4}\right)_{3}$ single crystals. ${ }^{53}$

\section{Piezoresponse hysteresis}

In addition to voltage and time dependencies of the domain formation and relaxation, we studied the switching behavior by applying a sequence of the voltage pulses for the tip stopped in the predetermined locations. Such studies are helpful for the understanding of switching mechanism and local defect structure under the tip. ${ }^{54-56}$ We observed that in the interior of the grain the loops are symmetric and have a well saturated shape typical for ferroelectric materials. However, on approaching the grain boundary, the shape of the hysteresis progressively changes, so that the negative part of the loop becomes distorted and effectively the negative remnant value of the effective piezoelectric coefficient $d_{33}$ vanishes close to the grain boundary. The variation of the remnant $d_{33}$ is shown in Fig. 8 as a function of distance. This feature can be a manifestation of the increased density of defects that has been shown to distort the shape of the hysteresis in the similar manner in $\mathrm{BiFeO}_{3}$ thin films. ${ }^{55} \mathrm{~A}$ monotonic decrease of the remnant $d_{33}$ indicates that the concentration of these defects can increase on approaching

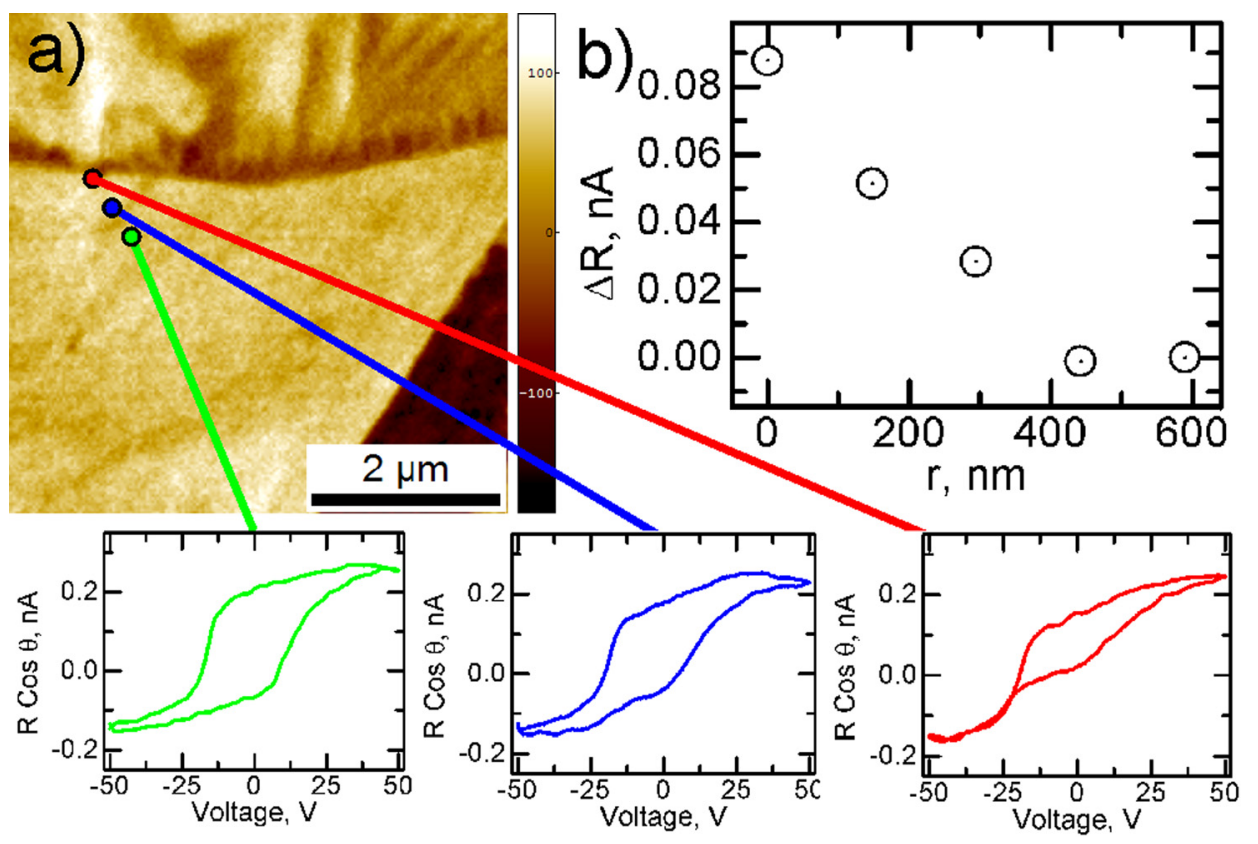

FIG. 8. (a) PFM images with marked hysteresis loops measured in the vicinity of the grain boundary and (b) negative remnant piezoresponse as a function of distance. 
the grain boundary and they are associated with the large enough electric fields that can severely distort the hysteresis. This observation is consistent with our data on domain imaging. The same reason (internal mechanical stress) can be responsible for the predominant domain twinning and hysteresis distortion close to the grain boundary. The correlation of the nanoscale piezoelectric properties with TEM imaging and defect investigation may bring further information about the peculiar properties of BCZT ceramics.

\section{CONCLUSIONS}

Piezoresponse Force Microscopy was used to study the complex domain structure in lead-free BCZT ceramics. The analysis of the histograms of piezoresponse images of the individual grains allowed to reveal multiple domain states including periodic lamella domains. This fact was attributed to possible phase co-existence in lead-free BCZT ceramics. The period of the domain structure scales with the subgrain size following well-known dependence observed in perovskite ceramics. Domain kinetics under the action of tip-induced electric field was studied with the final goal to understand the mechanism of the domain wall motion and interaction with defects and absorbate layer on the surface. Sufficiently, high domain wall velocity under localized poling can be responsible for the superior piezoelectric performance of BCZT ceramics. Domain relaxation was explained in terms of a polarization backswitching under the action of residual depolarization field exponentially decreasing with time due to bulk screening process. The distortion of the hysteresis loops near grain boundary was observed and attested to high concentration of defects and their interaction with localized field produced by the PFM tip.

\section{ACKNOWLEDGMENTS}

The equipment of Ural Center for Shared Use "Modern Nanotechnology" UrFU has been used. The research was made possible in part by the Ministry of Education and Science of the Russian Federation, (UID RFMEFI59414X0011), by RFBR (Grant Nos. 13-02-01391-a and 14-02-90447 Ukr-a), and by UrFU development program and under the joint supervision of doctorate thesis program of French government (MMR). This work was developed in the scope of the project CICECO-Aveiro Institute of Materials (Ref. FCT UID/CTM/ 50011/2013), financed by national funds through the FCT/ MEC and when applicable co-financed by FEDER under the PT2020 Partnership Agreement. I.C. would like to acknowledge the financial support from FCT, Portugal, through the Grant No. SFRH/BPD/81032/2011.

${ }^{1}$ B. Jaffe, W. R. Cook, and H. Jaffe, Piezoelectric Ceramics (Academic, New York, 1971).

${ }^{2}$ S. Trolier McKinstry and J. Muralt, Electroceramics 12, 717 (2004).

${ }^{3}$ S. P. Li, J. A. Eastman, Z. Li, C. M. Foster, R. E. Newnham, and L. E. Cross, Phys. Lett. A 212, 341346 (1996).

${ }^{4}$ T. M. Shaw, S. Trolier McKinstry, and P. C. McIntyre, Annu. Rev. Mater. Sci. 30, 263298 (2000).

${ }^{5}$ N. Nuraje, K. Su, A. Haboosheh, J. Samson, E. P. Manning, N. L. Yang, and H. Matsui, Adv. Mater. 18, 807811 (2006).
${ }^{6}$ D. D. Fong, G. B. Stephenson, S. K. Streiffer, J. A. Eastman, O. Auciello, P. H. Fuoss, and C. Thompson, Science 304, 16501653 (2004).

${ }^{7}$ J. Rodel, W. Jo, K. T. P. Seifert, E. Anton, T. Granzow, and D. Damianovic, J. Am. Ceram. Soc. 92, 11531177 (2009).

${ }^{8}$ I. Coondoo, N. Panwar, and A. L. Kholkin, J. Adv. Dielectr. 3, 1330002 (2013).

${ }^{9}$ S. J. Zhang, R. Xia, and T. R. Shrout, Appl. Phys. Lett. 91, 132913 (2007).

${ }^{10}$ J. G. Wu, D. Q. Xiao, Y. Y. Wang, W. J. Wu, B. Zhang, J. Li, and J. G. Zhu, Scr. Mater. 59, 750752 (2008).

${ }^{11}$ C. W. Ahn, H. C. Song, S. Nahm, S. Priya, S. H. Park, K. Uchino, H. G. Lee, and H. J. Lee, J. Am. Ceram. Soc. 89, 921925 (2006).

${ }^{12}$ Y. Saito, H. Takao, T. Tani, T. Nonoyama, K. Takatori, T. Homma, T. Nagaya, and M. Nakamura, Nature 432, 8487 (2004).

${ }^{13}$ A. J. Hatt and N. A. Spaldin, Phys. Rev. B 81, 054109 (2010).

${ }^{14}$ H. Liu, P. Yang, K. Yao, K. P. Ong, P. Wu, and J. Wang, Adv. Funct. Mater. 22, 937942 (2012).

${ }^{15}$ R. J. Zeches et al., Science 326, 977980 (2009).

${ }^{16} \mathrm{P}$. Hansen, D. Henning, and H. Schreinemacher, J. Am. Ceram. Soc. 81, 13691373 (1998).

${ }^{17}$ W. F. Liu and X. B. Ren, Phys. Rev. Lett. 103, 257602 (2009).

${ }^{18}$ H. X. Bao, C. Zhou, D. H. Xue, J. H. Gao, and X. B. Ren, J. Phys. D: Appl. Phys. 43, 465401 (2010).

${ }^{19}$ J. Gao, D. Xue, Y. Wang, D. Wang, L. Zhang, H. Wu, S. Guo, H. Bao, C. Zhou, W. Liu, S. Hou, G. Xiao, and X. Ren, Appl. Phys. Lett. 99, 092901 (2011).

${ }^{20}$ I. Coondoo, N. Panwar, H. Amorín, M. Alguero, and A. Kholkin, J. Appl. Phys. 113, 214107 (2013).

${ }^{21}$ D. Damjanovic, A. Biancoli, L. Batooli, A. Vahabzadeh, and J. Trodahl, Appl. Phys. Lett. 100, 192907 (2012).

${ }^{22}$ A. K. Tagantsev, L. E. Cross, and J. Fousek, Domains in Ferroic Crystals and Thin Films (Springer, New York, 2010).

${ }^{23}$ V. V. Shvartsman and A. L. Kholkin, J. Appl. Phys. 108, 042007 (2010).

${ }^{24}$ G. Arlt, D. Hennigs, and G. de With, J. Appl. Phys. 58, 16191625 (1985).

${ }^{25}$ G. Arlt, J. Mater. Sci. 25, 26552666 (1990).

${ }^{26}$ W. Cao and C. A. Randall, J. Phys. Chem. Solids 57, 14991505 (1996).

${ }^{27}$ S. Wada, S. Suzuki, T. Noma, T. Suzuki, M. Osada, M. Kakihana, S. E. Park, L. E. Cross, and T. R. Shrout, Jpn. J. Appl. Phys., Part 1 38, 55055511 (1999).

${ }^{28}$ G. Le Rhun, I. Vrejoiu, and M. Alexe, Appl. Phys. Lett. 90, 012908 (2007).

${ }^{29}$ I. Vrejoiu, G. Le Rhun, L. Pintilie, D. Hesse, M. Alexe, and U. Gosele, Adv. Mater. 18, 16571661 (2006).

${ }^{30}$ V. T. Tung, N. T. Tinh, T. V. Chuong, N. T. M. Huong, D. A. Tuan, and L. V. Truyen, J. Mod. Phys. 4, 16271631 (2013).

${ }^{31}$ P. Mishra and P. Kumar, Adv. Condens. Matter Phys. 2013, 858406 (2013).

${ }^{32}$ V. A. Zhirnov, Sov. Phys. JETP 8, 822 (1959).

${ }^{33}$ P. Marton, I. Rychetsky, and J. Hlinka, Phys. Rev. B 81, 144125 (2010).

${ }^{34}$ T. Tybell, P. Paruch, T. Giamarchi, and J. M. Triscone, Phys. Rev. Lett. 89, 097601 (2002).

${ }^{35}$ N. Pertsev, A. Petraru, I. Bdikin, D. Kiselev, and A. L. Kholkin, Nanotechnology 19, 375703 (2008).

${ }^{36}$ N. Pertsev, D. Kiselev, I. Bdikin, M. Kosec, and A. L. Kholkin, J. Appl. Phys. 110, 052001 (2011).

${ }^{37}$ A. L. Kholkin, I. K. Bdikin, V. V. Shvartsman, and N. A. Pertsev, Nanotechnology 18, 095502 (2007).

${ }^{38}$ M. Abplanalp, J. Fousek, and P. Gunter, Phys. Rev. Lett. 86, 57995802 (2001).

${ }^{39}$ T. Morita and Y. Cho, Appl. Phys. Lett. 84, 257259 (2004).

${ }^{40}$ S. Buhlmann, E. Colla, and P. Muralt, Phys. Rev. B 72, 214120 (2005).

${ }^{41}$ E. I. Shishkin, V. Ya. Shur, F. Schlaphof, and L. M. Eng, Appl. Phys. Lett. 88, 252902 (2006).

${ }^{42}$ V. Ya. Shur, A. V. Ievlev, E. V. Nikolaeva, E. I. Shishkin, and M. M. Neradovskiy, J. Appl. Phys. 110, 052017 (2011).

${ }^{43}$ B. L. Weeks, M. W. Vaughn, and J. J. Deyoreo, Langmuir 21, 80968098 (2005).

${ }^{44}$ A. Brugre, S. Gidon, and B. Gautier, J. Appl. Phys. 110, 052016 (2011).

${ }^{45}$ A. V. Ievlev, A. N. Morozovska, V. Ya. Shur, and S. V. Kalinin, Appl. Phys. Lett. 104, 092908 (2014).

${ }^{46}$ D. Dahan, M. Molotskii, G. Rosenman, and Y. Rosenwaks, Appl. Phys. Lett. 89, 152902 (2006).

${ }^{47}$ S. Kim, V. Gopalan, K. Kitamura, and Y. Furukawa, J. Appl. Phys. 90, 29492963 (2001). 
${ }^{48}$ L. Tian, V. Gopalan, and L. Galambos, Appl. Phys. Lett. 85, 44454447 (2004).

${ }^{49}$ M. I. Molotskii and M. M. Shvebelman, Philos. Mag. 85, 16371655 (2005).

${ }^{50}$ R. G. Batchko, V. Y. Shur, M. M. Fejer, and R. L. Byer, Appl. Phys. Lett. 75, 16731975 (1999).

${ }^{51}$ V. Ya. Shur, E. L. Rumyantsev, E. V. Nikolaeva, E. I. Shishkin, D. V. Fursov, R. G. Batchko, L. A. Eyres, M. M. Fejer, and R. L. Byer, Appl. Phys. Lett. 76, 143145 (2000).
${ }^{52}$ V. Ya. Shur, J. Mater. Sci. 41, 199210 (2006).

${ }^{53}$ V. Ya. Shur, Phase Transitions 65, 4972 (1998).

${ }^{54}$ A. Wu, P. M. Vilarinho, V. V. Shvartsman, G. Suchaneck, and A. L. Kholkin, Nanotechnology 16, 25872595 (2005).

${ }^{55}$ S. V. Kalinin, S. Jesse, B. J. Rodriguez, Y. H. Chu, R. Ramesh, E. A. Eliseev, and A. N. Morozovska, Phys. Rev. Lett. 100, 155703 (2008).

${ }^{56}$ D. Schrade, R. Mueller, D. Gross, T. Utschig, V. Ya. Shur, and D. C. Lupascu, Mech. Mater. 39, 161174 (2007). 